Coub-911111--25

PNL-SA--19319

DE92 006485

THE IMPACT OF SPECTRAL EFFECTS IN FAST

REACTORS ON DATA ANALYSIS AND

DEVELOPMENT OF FISSION-FUSION

CORRELATIONS

L. R. Greenwood

F. A. Garner

H. L. Heinisch

November 1991

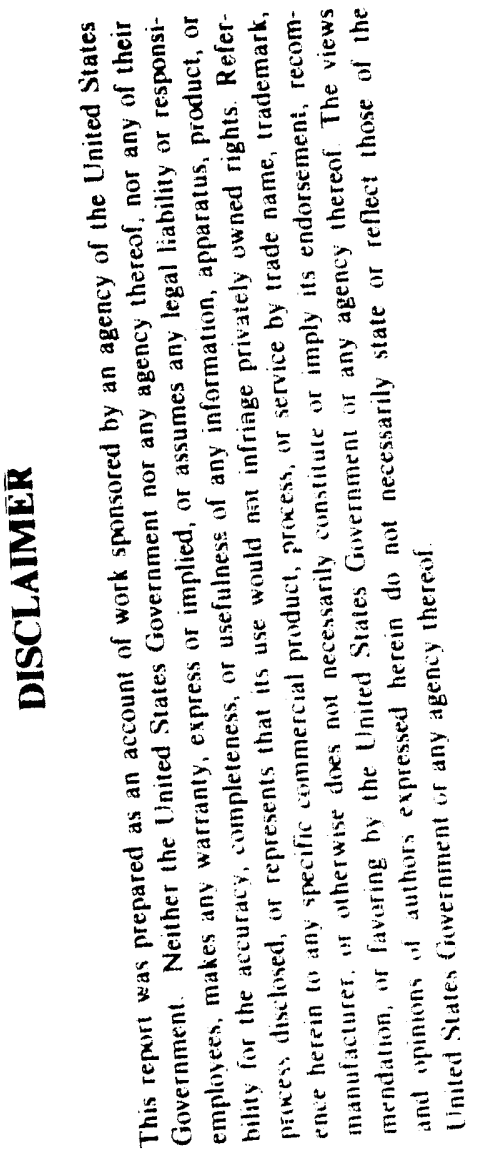

Presented at the

5 th International Conference on

Fusion Reactor Materials

November 17-22, 1991

Clearwater, Florida

Work supported by

the U.S. Department of Energy

under Contract DE-ACO6-76RLO 1830

Pacific Northwest Laboratory

Richland, Washington 99352

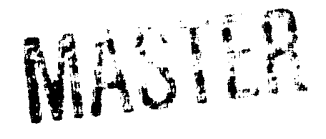




\title{
THE IMPACT OF SPECTRAL EFFECTS IN FAST REACTORS ON DATA ANALYSIS AND DEVELOPMENT OF FISSION-FUSION CORRELATIONS
}

\author{
L.R. Greenwood, F.A. Garner, and H.L. Heinisch
}

'Pacific Northwest Laboratory, P.0. Box 999, Richland, WA 99352, USA

\section{Abstract}

Models describing the production of freely migrating defects (FMD) during neutron irradiations were applied to materials studies in fast reactors, where neutron spectral effects have been largely ignored. Calculations for the Experimental Breeder Reactor II (EBR-II) and the Fast Flux Test Facility (FFTF) show that effects of spectral differences between in-core and out-ofcore positions are significantly larger on the basis of freely migrating, defects than on the basis of dpa. As an example, data on swelling behavior measured both in-core and out-of-core in EBR-II show nearly a linear dependence on FMD production but not on dpa.

\section{Introduction}

The development of radiation-resistant materials for fusion reactors relies on the correlation of test data obtained in a variety of neutron spectra, none of which are similar to those expected in fusion reactors. A successful correlation occurs when data are compared on the basis of a damage parameter that accounts for spectral differences. Displacements per atom (dpa) is a widtiy used and effective damage parameter, especially for neutron spectra in which a very large fraction of the damage is produced by high energy neutrons. As more is learned about the production of lattice defects in radiation damage events and the roles of defects in microstructural evolution, it is becoming clear that good correlations may require damage parameters based on more specific representations of defect production than is embodied in the dpa concept[1].

The embrittlement of fission reactor pressure vessels has been found to be substantially greater than predicted by tests conducted in-core and correlated with dpa as the exposure parameter[2]. Successful correlations of both in-core and pressure vessel data $[3,4,5]$ have recent 7 y been developed, however, and are based on the significant contributions to residual damage produced by thermal neutrons, which comprise a very large fraction of the neutrons at the pressure vesse1. The thermal neutron $(n, \gamma)$ reaction procuces energetic recoit atoms in iron near $400 \mathrm{eV}$, which are very efficient at producing mobile point defects. Correlations were achieved using energydependent production models of those defects that are assumed to be effective in changing the microstructure $[4,5]$. It should be noted that these were simple, preliminary models. The characterization of effective defects is still under investigation.

Another very promising concept, the production bias, incorporates the temperature dependence of interstitial and vacancy clusters against

-Pacific Northwest Labcratory is operated for the U.S. Department of Energy by Eattelle Memorial Institute under Contract DE-ACOE-76RLO 1830. 
dissolution and has been used to describe differences in the temperature dependence of the swelling rates for electron and neutron irradiations [6].

In this paper we examine the potential influence of the neutron energy dependence of the production of effective defects in fast neutron test facilities. Generally, dpa is considered a good damage parameter for correlating fast reactor data obtained in-core. However, current use of outof-core positions for spectral tailoring experiments requires that we assess the correlation of data from both in-core and out-of-core positions, where epithermal contributions to the spectrum become more important. Two current models of effective defect production are used to examine differences in the neutron spectral effects between in-core and out-of-core positions in both FFTF and EBR-II.

\section{Effective Defect Production}

Dpa is a calculated quantity that indicates the potential for creating point defects in a material exposed to a particular radiation source. It is based on a simple model, first proposed by Kinchin and Pease and modified by Norgett et al. [7], for partitioning the damage energy (that part of the recoil energy that goes into displacing atoms) by the amount required to produce a stable Frenkel pair. At very low recoil energies a few, isolated stable Frenkel pairs are produced in each event, about equal to the number predicted by the Kinchin-Pease model. At high recoil energies a collision cascade occurs, in which the damage energy is deposited in a small volume of the lattice. As the cascade energy dissipates, most of the displaced atoms recombine, leaving only very few point defects per unit damage energy. A significant fraction of the residual point defects are thought to be in clusters, whereas the remaining mobile defects are free to migrate away from the cascade region. The freely migrating defects and, depending on the temperature, those that dissociate from immobile clusters, are the point defects that effectively participate in microstructural changes.

Atomistic computer simulations [8] utilizing the binary collision cascade computer code MARLOWE [9] and stochastic annealing simulations yielded energy-dependent functions for the production of "freely migrating defects" in individual, isolated cascades at room temperature in $\mathrm{Cu}[10]$. The freely migrating interstitial function from reference 8 was chosen as a test case and is referred to as Hmod.

The second test case was an energy dependent function for freely migrating defects proposed by Wiedersich [11]. The function was fitted to Jata extracted from solute segregation experiments on $\mathrm{Ni}-\mathrm{Si}$ and $\mathrm{Cu}-\mathrm{Au}$ alloys irradiated at temperatures of 650-900 $\mathrm{K}$ with heavy ions [12]. This function is referred to as wmod. This model has had some success in describing materials effects for pressure vessel steel[4].

These preliminary models represent the scope of theoretical and experimental activities aimed at characterizing the production of the effertive defects in radiation damage. Both models have serious shortcomings. Hmod is based on computer modeling of the physical processes in individual cascades in a pure metal; it does not include the effects of intercascade interactions or alloy compositions. The empirical wmod function is fitted to 
data that are interpreted from the high damage rate experiments using rate theories that may not froperly represent defect production in casiades.

Relative to calculated dpa, both Hmod and Wmod are near unity at low energies and drop steeply with increasing energy to constant values of a few percent (see Figure 1). The primary difference between the two functions is the magnitude of the constant value reached at high energies. Wmod clearly predicts more dramatic spectral effects than Hmod because of the lower number of freely migrating defects at higher recoil energies.

\section{Calculational Method and Results}

Calculations were simplified by making use of the SPECTER computer code [13] which contains energy-dependent atomic recoil distributions for 40 different elements for neutron energies up to $20 \mathrm{MeV}$. A computer program was written to integrate the freely migrating defect models over the primary recoil energy distributions at each of 100 neutron energies between $1.0 \times 10^{-10}$ and $20 \mathrm{MeV}$. In this way, energy-dependent defect production cross sections were determined for each model. These cross sections were then added to the SPECTER cross section libraries so that defect production is calculated along with the standard dpa for any specified neutron energy spectrum and length of irradiation. The calculated cross sections are shown in Figure 2.

Defect calculations were performed as a function of axial location in rows 2 and 7 of EBR-II at Argonne National Laboratory, and for the Materials Open Test Assembly (MOTA) in FFTF at Hanford. Neutron spectra were taken from calculations for run $78 C$ in EBR-II [14] and for run cycle $9 A$ in FFTF [15]. In order to understand the effects of considering surviving defects as a damage parameter as opposed to dpa, the enhancement factor was determined for each axial location relative to midplane. The calculated dpa would thus have a va?ue of 1.0 at all locations. Note that the enhancements are only relative since, as is clearly shown in Figures 1 and 2, both Hmod and Wmod predict significantly fewer defects than the calculated number of displacements (dpa) on an absolute scale. As can be seen in Figures 3 and 4 , reiative defect production in the new models is enhanced by factors of 2 to 3 in comparison to dpa. This is a significant difference if one is attempting to correlate material property changes observed in irradiations conducted at various axial positions. Note that significant effects are seen for the below-core basket region and above-core levels 6-8 for the MOTA assembly in FFTF, positions that are being used more frequently in current fusion materials experiments.

\section{Comparisons with Materials Effects Data}

Many radiation-induced property changes are sensitive not only to crmposition but also to a wide array of environmental variables. Void swelling, for instance, is strongly sensitive to temperature history, displacement rate, and applied stress, a 71 of which exert their primary influence on the duration of the transient regime of swelling [16]. Temperature and displacement rate variations are frequently strongly coupled in many reactors, however, and displacement rate gradients are invariabiy accompanied by variations in neutron spectra [1]. This sometimes makes it difficult to separate the influence of each of these three variables. The 
potential impact of spectral variations in data analysis can be demonstrated using two published data sets.

Porter and Garner [17] examined the swelling of 1.5-m-long pressurized creep tubes constructed from annealed $304 \mathrm{~L}$ stainless steel that were irradiated in Row 7 of EBR-II to peak doses of $-80 \mathrm{dpa}$. Swelling was measured for ten one-inch sections from each pin; one at the core centerline, three just above the core bottom and six below the core bottom. The temperature varied from -380 to $-400^{\circ} \mathrm{C}$ over this range and the displacement rate varied by a factor of -4 . As shown in Figure 5, swelling along the length of these pins exhibited a complex behavior in response to variations in temperature, displacement rate and possibiy neutron spectra. If the Wmod enhancement factors calculated for Row 7 are used to normalize the data, however, much of the complexity disappears, particularly in the below-core region. Since the temperature difference is on $1 \mathrm{y} 10^{\circ} \mathrm{C}$ in this region, it is evident that the apparent sensitivity to displacement rate is much lower than previously assumed [16]. This is particularly true for highly stressed pins, where stress reduces the duration of the transient region and thereby its impact on the data analysis.

Swelling in the below-core region exhibits an almost linear dependence on FMD production. The maximum enhancement factor for this data set was calculated to be 2.0, using the Wmod model. Enhancement factors calculated with Hmod are smaller than with Wmod and hence are not as effective in linearizing the data. Wmod was similarly found to be more effective than Hmod in fitting pressure vessel steel data obtained in different irradiation facilities [4].

The impact of spectral enhancement factors is even easier to demonstrate for data sets where incubation-related phenomena are small, where the impact of temperature variations is minimal, and where a larger range of spectral variation is involved. Lewthwaite and Mosdale [18] provided such an opportunity when they reported an apparently strong inverse dependence of irradiation creep on displacement rate, as shown in Figure 6 . Using weighted helical springs suspended in sodium-filled tubes, spanning both in-core and deep below-core positions in the Dounreay Fast Reactor (DFR), they observed enhancement in creep rates as large as a factor of four for displacement rates varying as much as a factor of 20 . Lewthwaite and Mosedale concluded that there was "no unambiguous evidence" supporting a displacement flux effect on point defect recombination and suggested an alternative possibility involving "the inadequacy of the displaced atom concept" with its "incomplete description of events in energetic cascades." Although neutron spectral data were not available for the DFR reactor, the spectral effects are expected to be similar to those for EBR-II, as shown in Figure 4. It is interesting to note that the size of the spectral effects in Figure 6 is ciearly comparable to that in Figure 4, which suggests that the apparent displacement rate effect may well be more readily explained by spectral differences, with dpa rate effects playing a secondary role.

Spectral effectiveness damage calculations are also being performed for other reactors, including the Oak Ridge Research Reactor and the High Flux Isotopes Reactor, both at Oak Ridge National Laboratory. Besides pressure- 
vessel considerations mentionod previously, other data sets will be examined for spectral effects. The calculations will also be used to predict the possible magnitude of spectral effects for various reactor positions.

Spectral effects have been shown to be significant for fast reactors, especially in the comparison of data between in-core and out-of-core positions. Although the models considered in this paper appear to have some success in analyzing swelling data, as well as explaining apparent differences in pressure vessel steel data mentioned previously, it should be stressed that these models are preliminary attempts to describe complex phenomena. More work is needed to develop physically-based models and to test them in various neutron spectra.

\section{References}

1. F.A. Garner, H.L. Heinisch, R.L. Simons, and F.M. Mann, Radiation Effects and Defects in Solids, Vol. 113 (1990) 229-255.

2. Evaluation of HFIR Pressure-Vessel Integrity Considering Radiation Embrittlement, Eds. R.D. Cheverton, J.G. Merkie and R.K. Nanstad, ORNL/TM-10444 (1987).

3. L. K. Mansur and K. Farre11, J. Nucl. Mater 170 (1990) 236.

4. C.A. Hrabal, Modified Damage Parameters Applied to a Typical Light-Water Reactor's Pressure Vessel Supports, ASTM-Euratom Symposium on Reactor Dosimetry, Strasbourg, France, August 1990.

5. H.L. Heinisch, J. Nucl. Mater., 178 (1991) 19.

6. C.H. Woo, B.N. Singh, and F.A. Garner, these proceedings.

7. M.J. Norgett, M.T. Robinson and I.M. Torrens, Nucl. Eng. Des., 33 (1975) 50 .

8. H.L. Heinisch, J. Nucl. Mater., 117 (1983) 46.

9. M.T. Robinson and I.M. Torrens, Phys. Rev. B9, (1974) 5008.

10. H.L. Heinisch and F.M. Mann, J. Nucl. Mater., 122-123 (1984) 1023.

11. H. Wiedersich, J. Nucl. Mater., 179-181 (1991) 70.

12. L.E. Rehn and P.R. Okamoto, Mater. Sci. Forum 15-18 (1987) 985.

13. L.R. Greenwood and R.K. Smither, SPECTER: Neutron Damage Calculations for Materials Irradiations, ANL/FPP-TM-197 (1985).

14. F.C. Franklin, E.R. Ebersole, and R.R. Heinrich, Analys is of EBR-II LowPower Dosimetry Run 78C, ANL-77-76 (1977). 
15. R. L. Simons, Westinghouse Hanford Company, private communication (1990).

16. F. A. Garner, J. Nucl. Mater., 122-123(1984)459-47i.

17. D. L. Porter and F. A. Garner, ASTM STP 870,1985, pp. 212-220.

18. G. W. Lewthwaite and D. Mosedale, J. Nucl. Mater., 90(1980)205-215.

\section{Figure Captions}

1. Comparison of defect survivability fractions for the Hmod and Wmod models of freely migrating defects compared with the standard dpa model.

2. Comparison of displacement damage cross sections for iron for the Hmod and Wmod models with that for the standard dpa model.

3. Relative enhancements over the standard dpa model are shown for Hmod and Wmod as a function of axial location in the MOTA of FFTF.

4. Relative enhancements over the standard dpa model are shown for Hmod and Wmod as a function of axial location in row 7 of EBR-II.

5. (a) Swelling variations observed along the length of four annealed $304 \mathrm{~L}$ stainless steel tubes pressurized to different hoop stress levels and irradiated in EBR-II, plotted vs calculated displacement level. Effect of spectral effectiveness on these data is shown in (b) for a stressfree pin and (c) for a highly stressed pin, plotted vs FMD.

6. Enhancement of creep rates measured by Lewthwaite and Mosedale in various positions in and below the core of the DFR reactor relative to that measured at core center, implying a strong dependence on either displacement rate or neutron spectra. 
Freely Migrating Defects

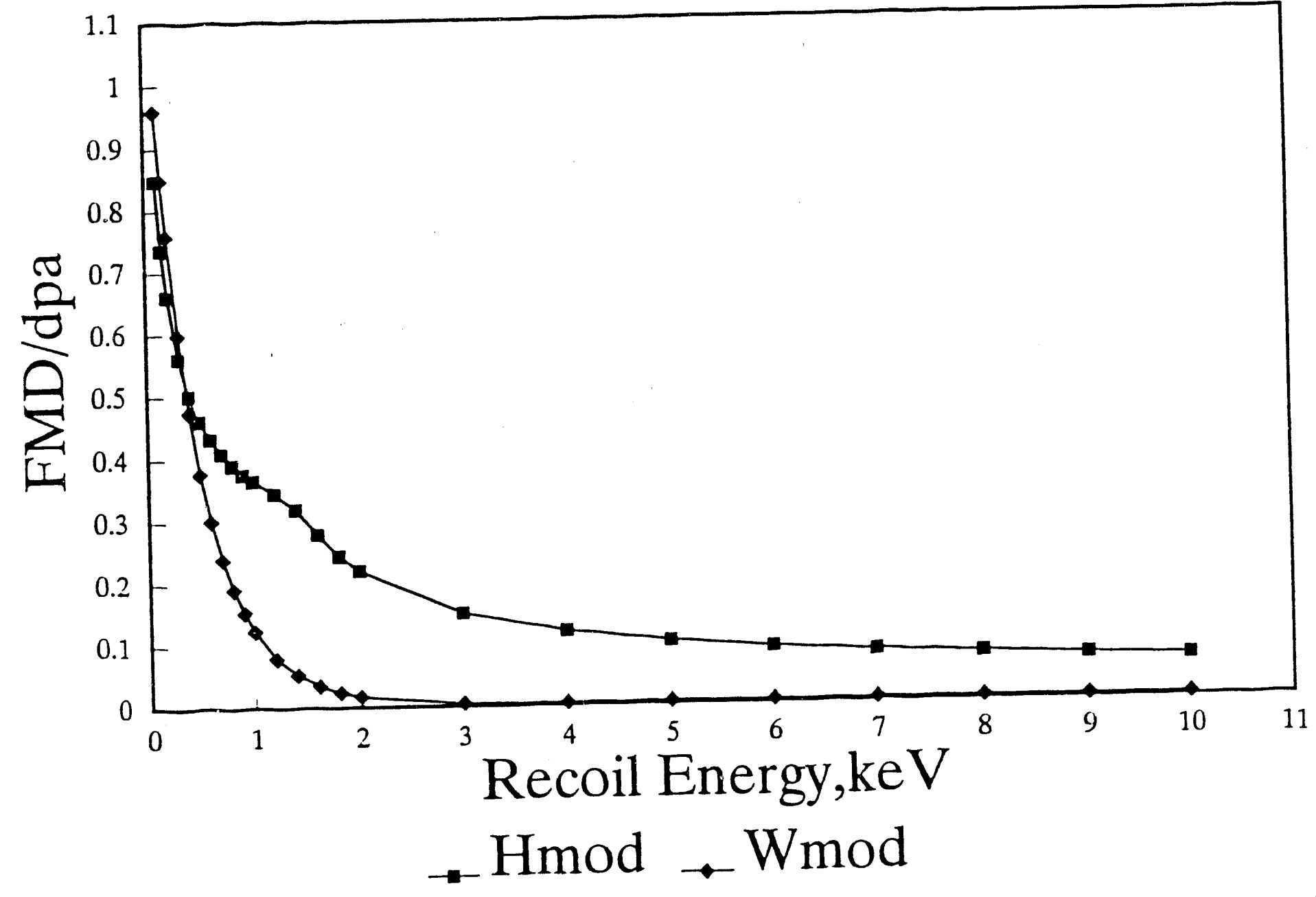




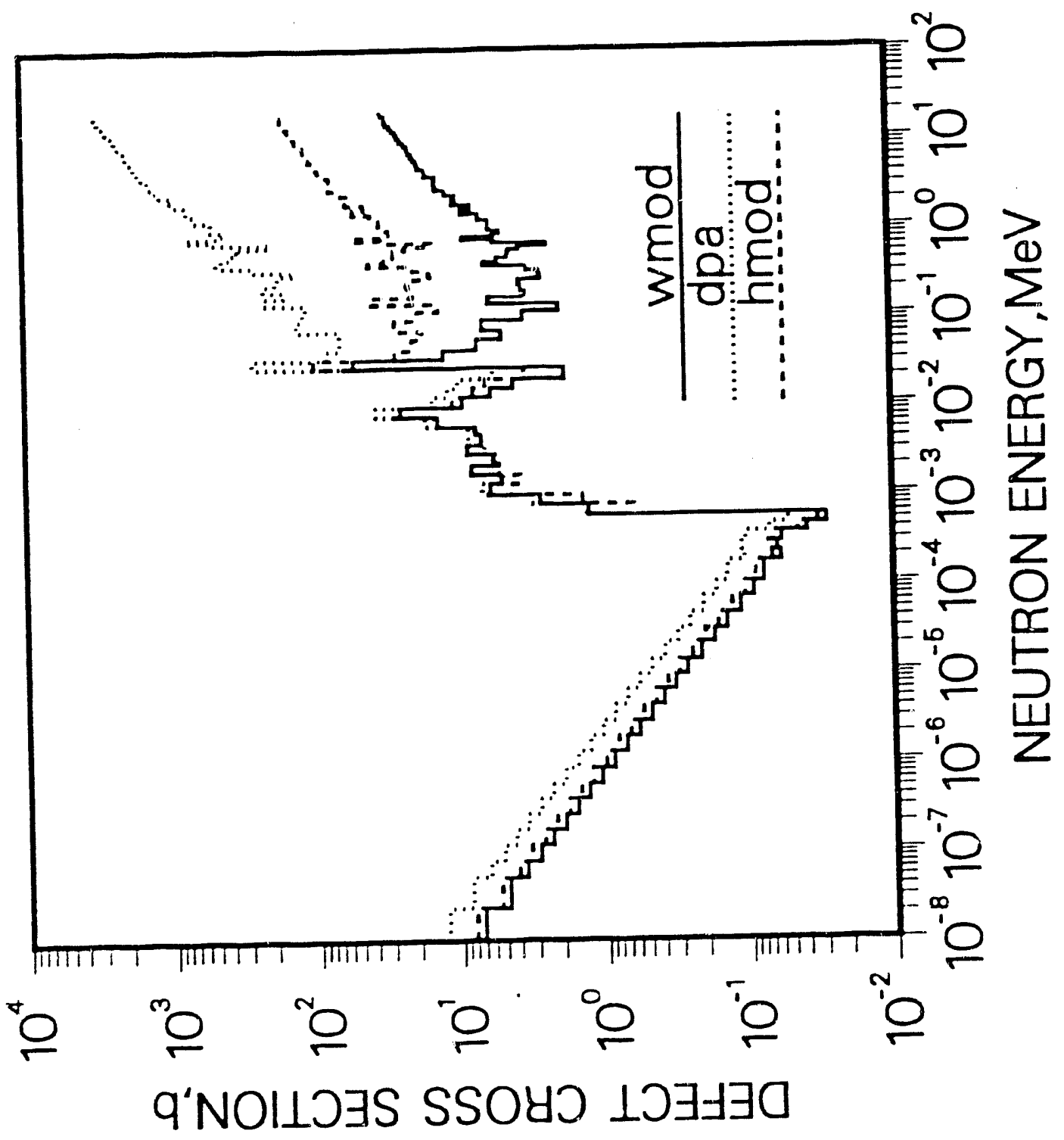


FFTF 9A

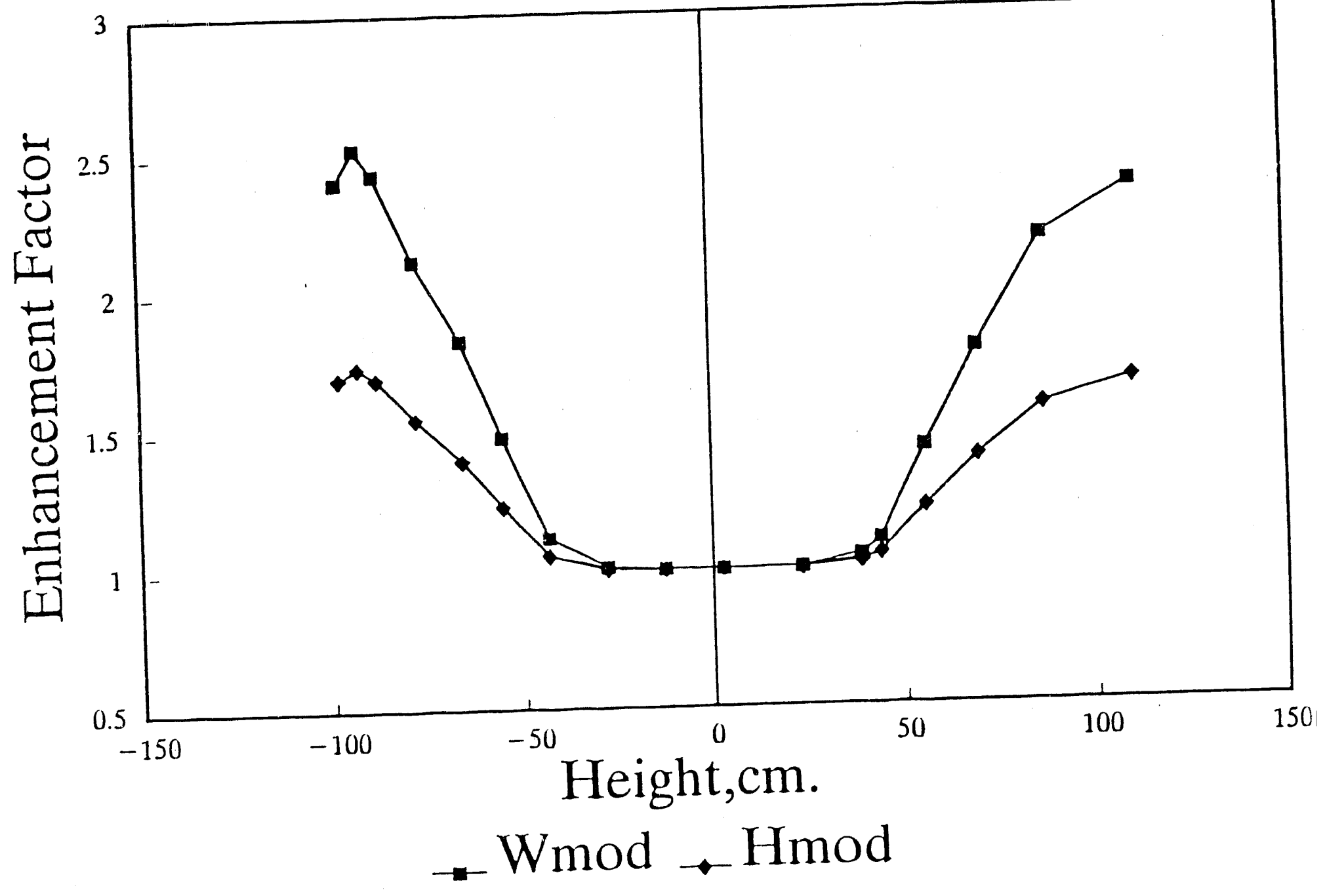

(3) 
EBR II Row 7

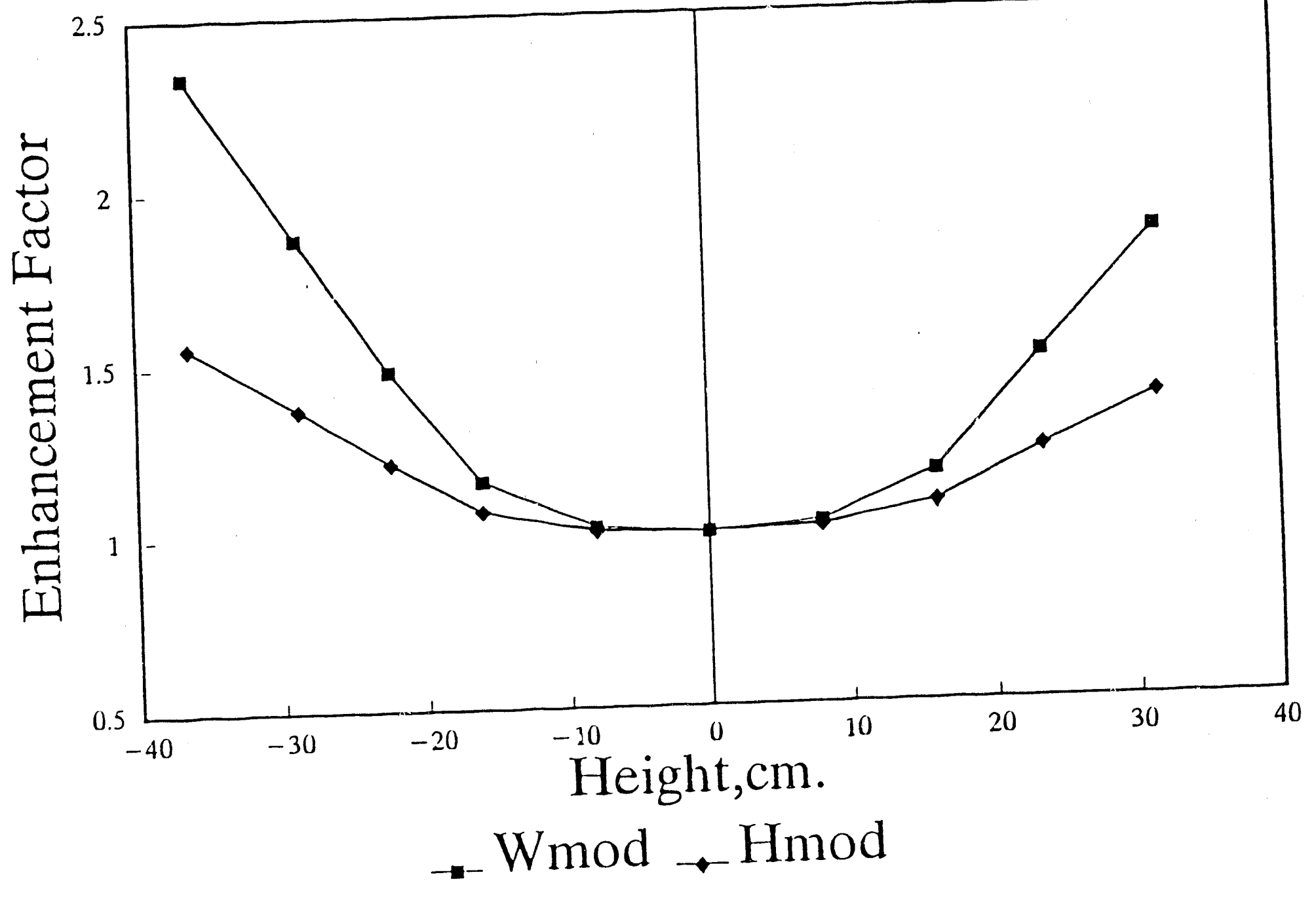




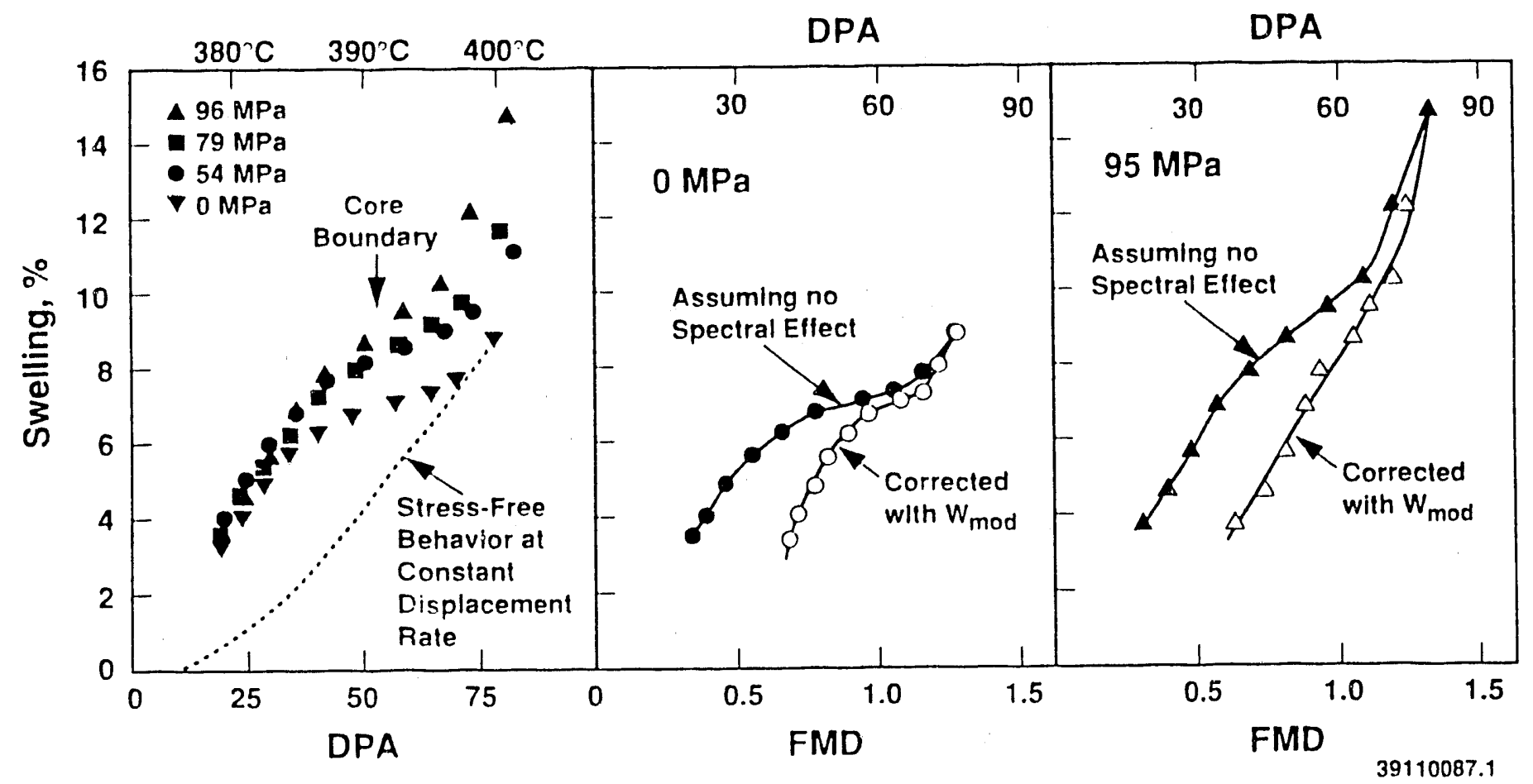



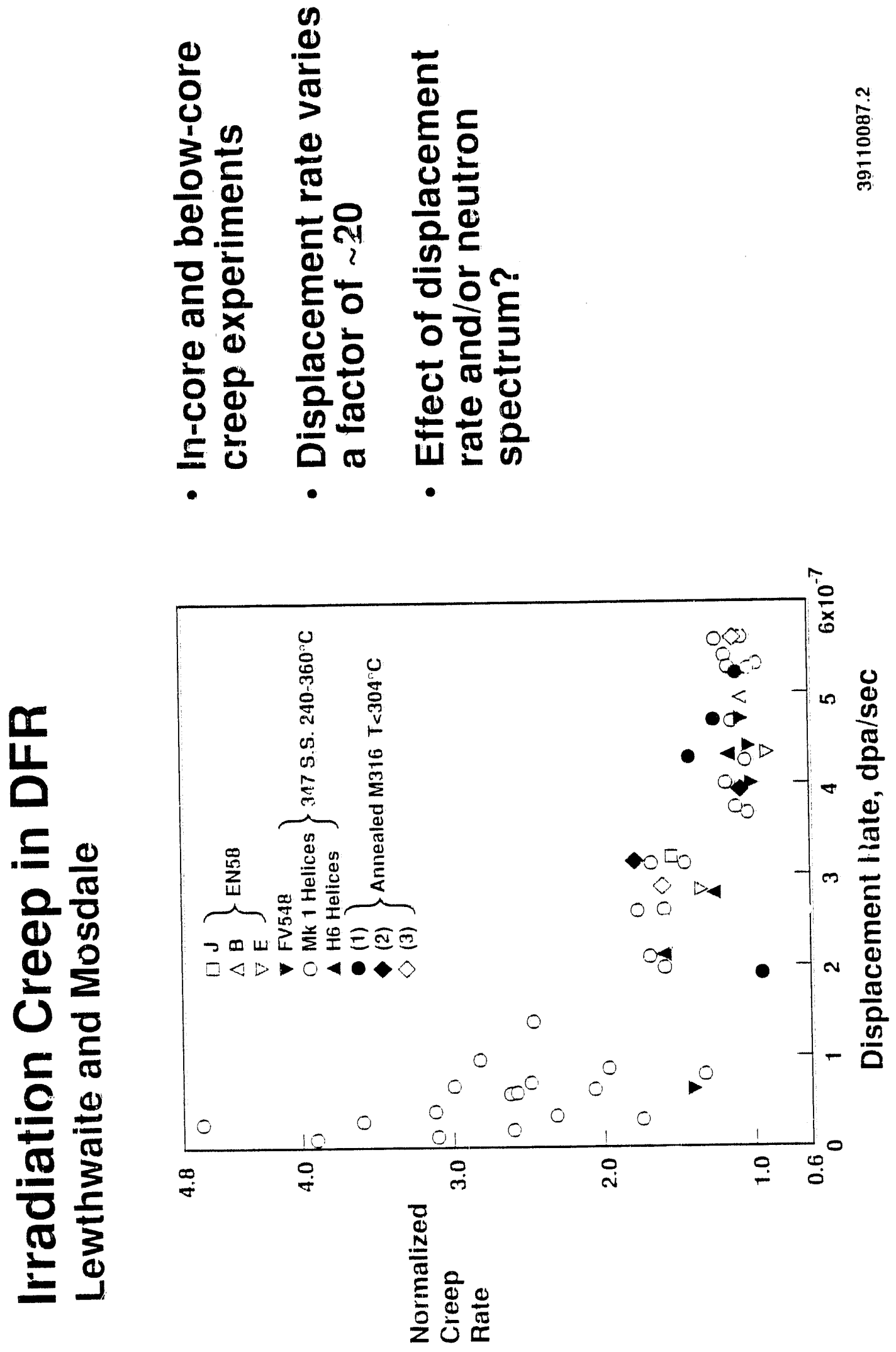


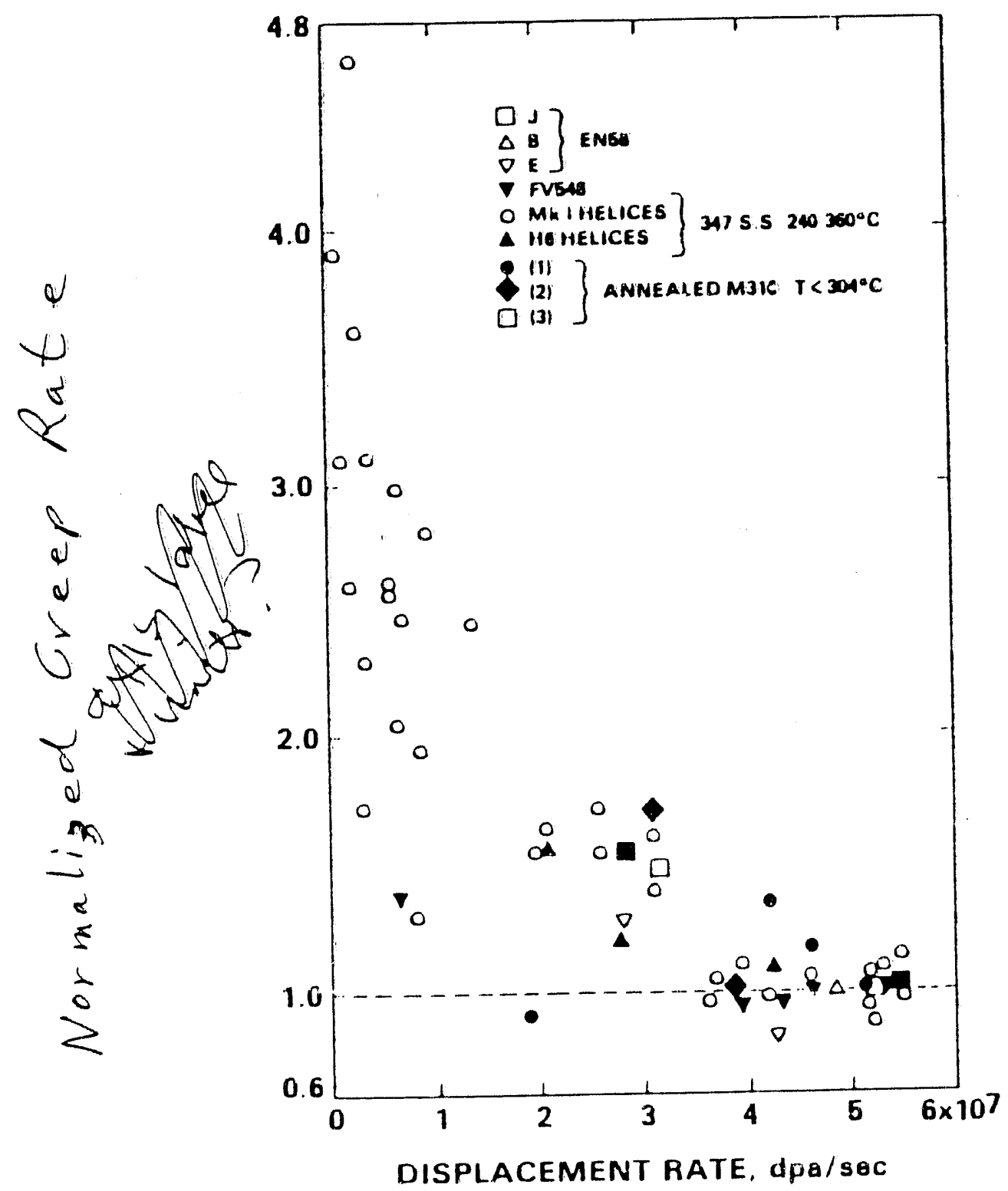

(6)

Heol wal IIt J 

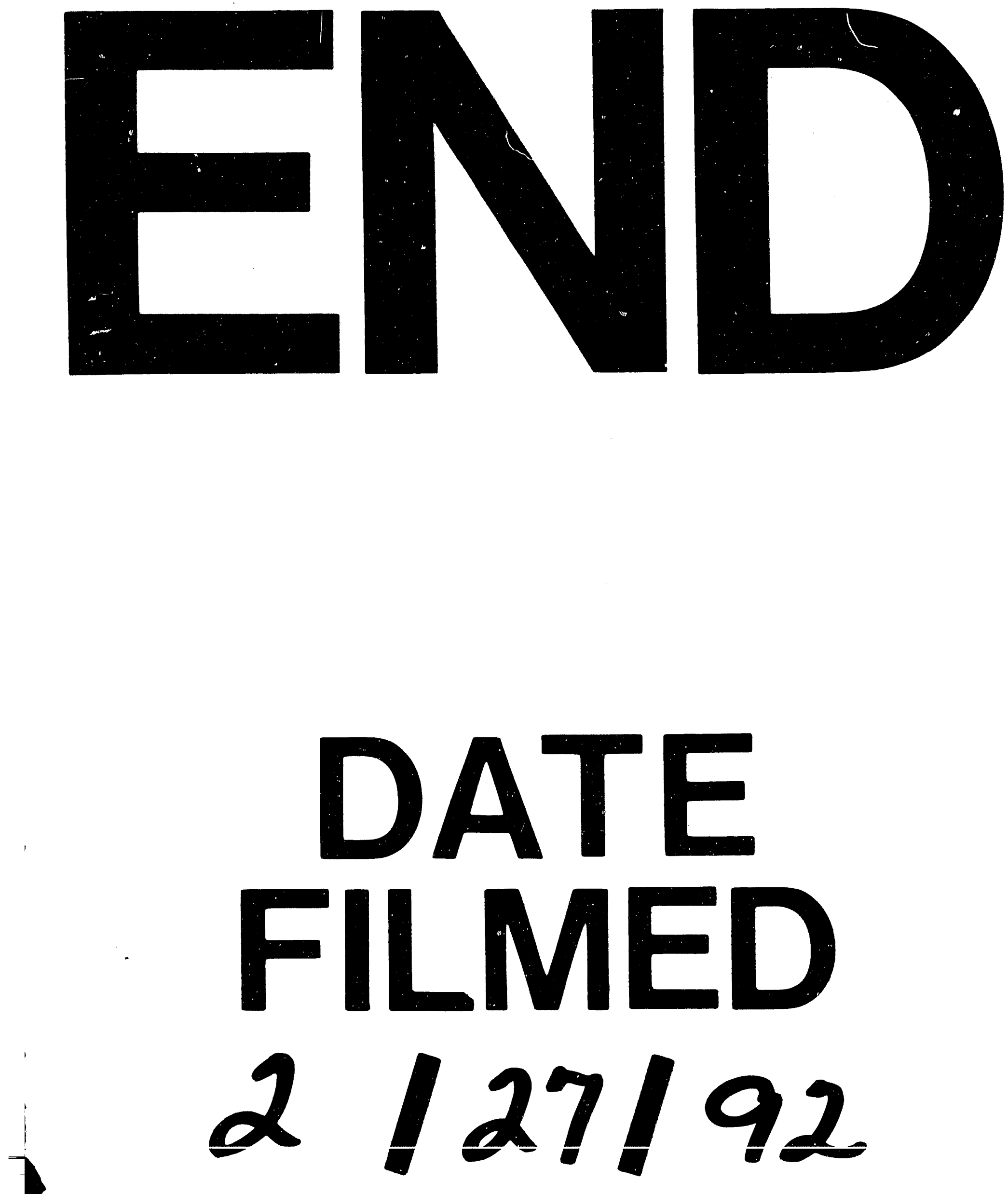
